\title{
Polypharmacy in older people: time to take action
}

\author{
Marta Gutiérrez-Valencia ${ }^{1} \cdot$ Nicolas Martínez-Velilla $^{2} \cdot$ Arturo Vilches-Moraga $^{3}$ (i)
}

Received: 8 November 2018 / Accepted: 20 November 2018 / Published online: 30 November 2018

(c) European Geriatric Medicine Society 2018

Keywords Polypharmacy $\cdot$ Medication reconciliation $\cdot$ Older people $\cdot$ Frailty $\cdot$ Multidisciplinary $\cdot$ Comprehensive geriatric assessment $\cdot$ Holistic $\cdot$ Transition of care

Medication is the most used medical intervention to improve health. However, in the older patient the prescription of medication becomes a complex task that poses a challenge for the clinician. Changes in pharmacokinetics and pharmacodynamics associated with age, the exclusion of older patients in clinical trials and their consequent underrepresentation in clinical practice guidelines, high multimorbidity and other factors that influence the prognosis and management of these patients, such as frailty, dementia or limited life expectancy, will condition the use of medications in older patients. Polypharmacy is highly prevalent in this population, due to the accumulation of chronic diseases and the presence of multiple prescribers. The important problems resulting from this polypharmacy are already well known, due to its association with an increased risk of adverse effects, drug-drug interactions, poor treatment adherence, more hospitalizations, higher mortality and increased costs [1].

Population aging leads to a progressive increase in older patients in all healthcare settings and medical specialties. Knowing the complexity and risks associated with polypharmacy, it is the responsibility of all professionals to be involved in the adequate care of the older patient and the proper management of their medication. There has been

Arturo Vilches-Moraga

avilchesmoraga@gmail.com

1 Pharmacy Department, Navarrabiomed, Universidad Pública de Navarra (UPNA), Complejo Hospitalario de Navarra (CHN), IdiSNA, Pamplona, Navarra, Spain

2 Department of Geriatric Medicine, Navarrabiomed, Universidad Pública de Navarra (UPNA), Complejo Hospitalario de Navarra (CHN), IdiSNA, Pamplona, Navarra, Spain

3 Ageing and Complex Medicine Directorate, Salford Royal NHS Foundation Trust, Faculty of Medical and Human Sciences, University of Manchester, Salford, UK progress in recent years in this regard, but it is time for all of us to take action; what is the next step?

From a global perspective, given the complexity and differential characteristics in older patients, we must take into account the paradigm shift of the end of the disease era: medical care focused on the diagnosis and treatment of individual diseases is "at best out of date and at worst harmful". The time has come to abandon the disease as the primary focus of medical care and to place frailty and function at the centre of care models [2]. This idea is no longer a theory, but a real and pressing need. In the same way, polypharmacy and the associated negative effects are no more than a consequence of the traditional model of care based on a disease-centred approach. Aging will occupy increasingly wider and relevant positions in most disciplines and medical specialties, which makes it necessary for all of them to begin to take this change of vision, and to be impregnated with some geriatric concepts, such as frailty and comprehensive geriatric assessment. This, of course, must be translated into one of the main tools for the prevention and improvement of health: medicines. Beyond the acute or chronic pathology on which we want to intervene, we must look at the whole picture, since all factors are related: not only the patients' antecedents and medical problems, but also their functional, cognitive, affective or social situation, their geriatric syndromes, the rest of its pharmacological and non-pharmacological treatment, the patients' expectations, their frailty status and quality of life, and the individual risks and therapeutic goals. As an example, frailty and pre-frailty have shown to be associated with polypharmacy [3]. In older persons, it is particularly relevant that we consider polypharmacy before prescribing a drug agent. It is imperative that we take into account drug-drug interactions, therapeutic cascades, frequent adverse effects (such as falls or delirium) or the potentially inappropriate medications. Other objectives of 
adequate drug prescribing include identification of medication risks and adverse effects, prioritize the most important problems and make treatment targets more flexible. It is also necessary to keep in mind that patients may have different preferences, attitudes to medicines, experience of burden of treatment and own therapeutic goals, and they have the right to be involved in decision making on drug treatment.

Knowing how to analyse all this information adequately and integrate it to include it in decision making on medication prescribing is a complex task that requires investing time, continuously updating knowledge and improving teamwork. The multidisciplinary work must be an essential piece when addressing polypharmacy and the appropriate use of medications in older adults. Even being aware and being prepared for the challenges of polypharmacy and the prescription of medicines in older adults, in many cases the collaboration of specialized professionals will be beneficial. Two clear examples would be the participation of a geriatrician who makes a geriatric comprehensive assessment and conducts an individualized therapeutic plan according to the needs of the patient, and a clinical pharmacist, who can make an exhaustive medication review and advice on pharmacological aspects and medication adherence [4].

In cases where patient management is carried out without the collaboration of these professionals, it would be advisable to at least know certain tools that can help us make better decisions about drug prescribing, and include them in our daily practice, our computer systems, etc. The main instruments to optimize pharmacological treatment in the older patients have been classically divided into explicit tools, which are lists based on the criteria of potentially inappropriate medications in older people such as the STOPP/ START criteria [5], and implicit criteria and tools, based on clinical judgments, which propose simple questionnaires or algorithms to help improving medication appropriateness [6]. In addition, as the number of medications is the most significant predictor of the inappropriate use of drugs, deprescription has been proposed as a possible solution to address this problem. It has been described as the process of withdrawal of an inappropriate medication, supervised by a health care professional with the goal of managing polypharmacy and improving outcomes [7]. Other recent tools have focused on the adequate use of medications in older patients with limited life expectancy or at the end of life, situations that can be especially complex $[8,9]$.

Finally, we must pay attention to the problems of medication reconciliation derived from the transitions of care, like hospital admission and discharge or nursing home admission, and the presence of different prescribers. Medication reconciliation is the process of obtaining and documenting a complete and accurate list of current patient medications and comparing this list with medication orders at each point of care transition to identify and rectify any discrepancies before patient harm occurs, and should be implemented in transitions of care, especially in patients with polypharmacy [10]. Processes and tools should also be adapted for this purpose [11].

In summary, the management of polypharmacy in older patients involves a paradigm shift that abandons the disease as the axis of health care towards an integral approach linked to multidisciplinary work, dedication and specific preparation for medication prescribing in these patients, and a focus on medication reconciliation in transitions of care. Knowing how to face the challenge of polypharmacy and its consequences for patients, healthcare systems and society is everyone's responsibility and its success will depend on the involvement of all professionals.

\section{Compliance with ethical standards}

Conflict of interest The authors declare that we have no conflict of interest.

Ethical approval All procedures performed in studies involving human participants were in accordance with the ethical standards of the institutional and/or national research committee and with the 1964 Helsinki declaration and its later amendments or comparable ethical standards.

Informed consent Informed consent was obtained from all individual participants included in the study.

\section{References}

1. Maher RL, Hanlon J, Hajjar ER (2014) Clinical consequences of polypharmacy in elderly. Expert Opin Drug Saf 13(1):57-65. https ://doi.org/10.1517/14740338.2013.827660

2. Cesari M, Marzetti E, Thiem U, Pérez-Zepeda MU, Abellan Van Kan GA, Landi F, Petrovic M, Cherubini A, Bernabei R (2016) The geriatric management of frailty as paradigm of "The end of the disease era". Eur J Intern Med 31:11-14. https://doi.org/10.1016/j. ejim.2016.03.005

3. Palmer K, Villani ER, Vetrano DL, Cherubini A, Cruz-Jentoft AJ, Curtin D et al (2018) Association of polypharmacy and hyperpolypharmacy with frailty states: a systematic review and meta-analysis. Eur Geriatr Med. https://doi.org/10.1007/s41999-018-0124-5

4. Walgraeve K, Van der Linden L, Flamaing J, Fagard K, Spriet I, Tournoy $J(2018)$ Feasibility of optimizing pharmacotherapy in heart failure patients admitted to an acute geriatric ward: role of the clinical pharmacist. Eur Geriatr Med 9:103-111

5. O'Mahony D, O'Sullivan D, Byrne S, O'Connor MN, Ryan C, Gallagher P (2015) STOPP/START criteria for potentially inappropriate prescribing in older people: version 2. Age Ageing 44:213-218

6. Zullo AR, Gray SL, Holmes HM, Marcum ZA (2018) Screening for medication appropriateness in older adults. Clin Geriatr Med 34:39-54

7. Reeve E, Gnjidic D, Long J, Hilmer S (2015) A systematic review of the emerging definition of 'deprescribing' with network analysis: implications for future research and clinical practice. Br J Clin Pharmacol 80:1254-1268

8. Lavan AH, Gallagher P, Parsons C, O'Mahony D (2017) STOPPFrail (Screening Tool of Older Persons Prescriptions in Frail adults 
with limited life expectancy): consensus validation. Age Ageing 46:600-607

9. Morin L, Laroche ML, Vetrano DL, Fastbom J, Johnell K (2018) Adequate, questionable, and inadequate drug prescribing for older adults at the end of life: a European expert consensus. Eur J Clin Pharmacol. https://doi.org/10.1007/s00228-018-2507-4

10. Lehnbom EC, Stewart MJ, Manias E, Westbrook JI (2014) Impact of medication reconciliation and review on clinical outcomes. Ann Pharmacother 48:1298-1312
11. Dumur J, Chassagne P, Gbaguidi X et al (2018) Using a structured reconciliation medication form improves medication transition from hospital to community care and primary care physicians' adherence with medication adaptations and recommendations. Eur Geriatr Med. https://doi.org/10.1007/s41999-018-0125-4 